\title{
ON THE RATIONALITY OF POINCARÉ SERIES OF GORENSTEIN ALGEBRAS VIA MACAULAY'S CORRESPONDENCE
}

\author{
GIANFRANCO CASNATI, JOACHIM JELISIEJEW AND ROBERTO NOTARI
}

ABSTRACT. Let $A$ be a local Artinian Gorenstein algebra with maximal ideal $\mathfrak{M}$,

$$
P_{A}(z):=\sum_{p=0}^{\infty}\left(\operatorname{Tor}_{p}^{A}(k, k)\right) z^{p}
$$

its Poicaré series. We prove that $P_{A}(z)$ is rational if either $\operatorname{dim}_{k}\left(\mathfrak{M}^{2} / \mathfrak{M}^{3}\right) \leq 4$ and $\operatorname{dim}_{k}(A) \leq 16$, or there exist $m \leq 4$ and $c$ such that the Hilbert function $H_{A}(n)$ of $A$ is equal to $m$ for $n \in[2, c]$ and equal to 1 for $n=c+1$. The results are obtained due to a decomposition of the apolar ideal $\operatorname{Ann}(F)$ when $F=G+H$ and $G$ and $H$ belong to polynomial rings in different variables.

1. Introduction and notation. Throughout the whole paper, $k$ will denote an algebraically closed field of characteristic 0. By ring (respectively, $k$-algebra) we mean a Noetherian, associative, commutative and unitary $\operatorname{ring} A$ (respectively, algebra $A$ over $k$ ). When $A$ is local we will denote by $\mathfrak{M}$ its maximal ideal and we will assume that $k:=A / \mathfrak{M}$.

Since J.P. Serre asked in 1955 if the Poincaré series of the local ring $A$, i.e.,

$$
P_{A}(z):=\sum_{p=0}^{\infty} \operatorname{dim}_{k}\left(\operatorname{Tor}_{p}^{A}(k, k)\right) z^{p}
$$

2010 AMS Mathematics subject classification. Primary 13D40, Secondary $13 \mathrm{H} 10$.

Keywords and phrases. Artinian Gorenstein local algebra, rational Poincaré series.

The first and third authors are supported by the framework of PRIN 2010/11 "Geometria delle varietà algebriche," cofinanced by MIUR. The second author is supported by the project, "Secant varieties, computational complexity, and toric degenerations" realized within the Homing Plus programme of Foundation for Polish Science, co-financed from European Union, Regional Development Fund. This paper is a part of "Computational complexity, generalized Waring type problems and tensor decompositions" project within "Canaletto," the executive program for scientific and technological cooperation between Italy and Poland, 2013-2015.

Received by the editors on July 4, 2013, and in revised form on June 3, 2014. 
is rational, also proving its rationality when $A$ is regular, many authors have proved results confirming the rationality of $P_{A}$ in several cases.

Thereafter, in 1982, D.J. Anick gave the first example of an Artinian local algebra $A$ with transcendental $P_{A}$. Shortly afterwards, in 1983, the existence of an Artinian, Gorenstein, local ring with $\mathfrak{M}^{4}=0$ and transcendental $P_{A}$ was proved by $\mathrm{R}$. Bøgvad.

Nevertheless, several results show that large classes of local rings $A$ have rational Poincaré series, e.g., J. Tate proved the rationality for complete intersections rings (see [16]), L. Avramov, A. Kustin, M. Miller and C. Jacobsson, A. Kustin and M. Miller for Gorenstein local rings with $\operatorname{dim}_{k}\left(\mathfrak{M} / \mathfrak{M}^{2}\right) \leq 4$ (see $[\mathbf{1}, \mathbf{1 3}]$ respectively), J. Sally and J. Elias, G. Valla for Gorenstein local rings with $\operatorname{dim}_{k}\left(\mathfrak{M}^{2} / \mathfrak{M}^{3}\right) \leq 2$ (see $[\mathbf{8}, \mathbf{1 4}]$, respectively), the authors for Gorenstein local rings of multiplicity at most 10 (see [5]), the authors, jointly with J. Elias and M.E. Rossi for Gorenstein local $k$-algebras with $\operatorname{dim}_{k}\left(\mathfrak{M}^{2} / \mathfrak{M}^{3}\right)=4$ and $\mathfrak{M}^{4}=0$ (see [4]).

All of the above results are based on the same smart combination of results on the Poincaré series due to L. Avramov and G. Levin (see [2]) and T.H. Gulliksen and G. Levin (see [11]) first used in [14] combined with suitable structure results on Gorenstein rings and $k$ algebras. In this last case, a fundamental role has been played by Macaulay's correspondence.

In Section 2, we give a quick resumé of the main results that we need later on in the paper about Macaulay's correspondence. In Section 3, we extend to arbitrary $k$-algebras a very helpful decomposition result already used in a simplified form by J. Elias and M.E. Rossi in [7] and in the aforementioned [4] for $k$-algebras with $\mathfrak{M}^{4}=0$. In Section 4, we explain how to relate the rationality of the Poincaré series of Gorenstein $k$-algebras with their representation in the setup of Macaulay's correspondence making use of the aforementioned decomposition result. Finally, in Section 5, we use that relationship in order to prove the following two results generalizing the aforementioned quoted results.

Theorem A. Let $A$ be an Artinian, Gorenstein local k-algebra with maximal ideal $\mathfrak{M}$. If there are integers $m \leq 4$ and $c \geq 1$ such that 


$$
\operatorname{dim}_{k}\left(\mathfrak{M}^{t} / \mathfrak{M}^{t+1}\right)= \begin{cases}m & \text { if } t=2, \ldots, c \\ 1 & \text { if } t=c+1\end{cases}
$$

then $P_{A}$ is rational.

Theorem B. Let $A$ be an Artinian, Gorenstein local $k$-algebra with maximal ideal $\mathfrak{M}$. If $\operatorname{dim}_{k}\left(\mathfrak{M}^{2} / \mathfrak{M}^{3}\right) \leq 4$ and $\operatorname{dim}_{k}(A) \leq 16$, then $P_{A}$ is rational.

1.1. Notation. For each $N \in \mathbb{N}$ we set $S[N]:=k\left[\left[x_{1}, \ldots, x_{N}\right]\right]$ and $P[N]:=k\left[y_{1}, \ldots, y_{N}\right]$. We denote by $S[N]_{q}$ (respectively, $P[N]_{q}$ ) the homogeneous component of degree $q$ of such a graded $k$-algebra, and we set

$$
S[N]_{\leq q}:=\bigoplus_{i=1}^{q} S[N]_{i}
$$

respectively,

$$
P[n]_{\leq q}:=\bigoplus_{i=1}^{q} P[n]_{i}
$$

Finally, we set $S[n]_{+}:=\left(x_{1}, \ldots, x_{n}\right) \subseteq S[n]$. The ideal $S[n]_{+}$is the unique maximal ideal of $S[N]$.

A local ring $R$ is Gorenstein if its injective dimension as an $R$-module is finite.

If $\gamma:=\left(\gamma_{1}, \ldots, \gamma_{N}\right) \in \mathbb{N}^{N}$ is a multi-index, then we set $t^{\gamma}:=$ $t_{1}^{\gamma_{1}} \cdots t_{N}^{\gamma_{N}} \in k\left[t_{1}, \ldots, t_{N}\right]$.

For all other notation and results we refer to [10].

2. Preliminary results. In this section we list the main results on $k$-algebras we need in the next sections. Let $A$ be a local, Artinian $k$-algebra with maximal ideal $\mathfrak{M}$. We denote by $H_{A}$ the Hilbert function of the graded associated algebra

$$
\operatorname{gr}(A):=\bigoplus_{t=0}^{+\infty} \mathfrak{M}^{t} / \mathfrak{M}^{t+1}
$$

We know that

$$
A \cong S[n] / J
$$


for a suitable ideal $J \subseteq S[n]_{+}^{2} \subseteq S[n]$, where $n=\operatorname{emdim}(A):=H_{A}(1)$. Recall that the socle degree $\operatorname{sdeg}(A)$ of $A$ is the greatest integer $s$ such that $\mathfrak{M}^{s} \neq 0$.

We have an action of $S[n]$ over $P[n]$ given by partial derivation defined by identifying $x_{i}$ with $\partial / \partial y_{i}$. Hence,

$$
x^{\alpha} \circ y^{\beta}:= \begin{cases}\alpha !\left(\begin{array}{l}
\beta \\
\alpha
\end{array}\right) y^{\beta-\alpha} & \text { if } \beta \geq \alpha, \\
0 & \text { if } \beta \geq \alpha .\end{cases}
$$

Such an action endows $P[n]$ with a structure of the module over $S[n]$. If $J \subseteq S[n]$ is an ideal and $M \subseteq P[n]$ is an $S[n]$-submodule we set

$$
\begin{aligned}
J^{\perp} & :=\{F \in P[n] \mid g \circ F=0, \text { for all } g \in J\}, \\
\operatorname{Ann}(M) & :=\{g \in S[n] \mid g \circ F=0, \text { for all } F \in M\} .
\end{aligned}
$$

For the following results, see e.g., the paper [9] by J. Emsalem, the book [12] by A. Iarrobino and the references therein. Macaulay's inverse system theorem is based on the fact that constructions $J \mapsto J^{\perp}$ and $M \mapsto \operatorname{Ann}(M)$ give rise to an inclusion-reversing bijection between ideals $J \subseteq S[n]$ such that $S[n] / J$ is a local Artinian $k$-algebra and finitely generated $S[n]$-submodules $M \subseteq P[n]$. In this bijection, Gorenstein $k$-algebras $A$ with $\operatorname{sdeg}(A)=s$ correspond to cyclic $S[n]$ submodules $\langle F\rangle_{S[n]} \subseteq P[n]$ generated by a polynomial $F$ of degree $s$. We simply write $\operatorname{Ann}(F)$ instead of $\operatorname{Ann}\left(\langle F\rangle_{S[n]}\right)$.

On the one hand, given an $S[n]$-module $M$, we define

$$
\operatorname{tdf}(M)_{q}:=\frac{M \cap P[n]_{\leq q}+P[n]_{\leq q-1}}{P[n]_{\leq q-1}}
$$

where

$$
P[n]_{\leq q}:=\bigoplus_{i=0}^{q} P[n]_{i},
$$

and

$$
\operatorname{tdf}(M):=\bigoplus_{q=0}^{\infty} \operatorname{tdf}(M)_{q} .
$$

The module $\operatorname{tdf}(M)$ can be interpreted as the $S[n]$-submodule of $P[n]$ generated by the top degree forms of all polynomials in $M$. 
On the other hand, for each $f \in S[n]$, the lowest degree of monomials appearing with non-zero coefficient in the minimal representation of $f$ is called the order of $f$ and it is denoted by ord $(f)$. If

$$
f=\sum_{i=\operatorname{ord}(f)}^{\infty} f_{i}, \quad f_{i} \in S[n]_{i}
$$

then $f_{\text {ord }(f)}$ is called the lower degree form of $f$. It will be denoted in what follows with $\operatorname{ldf}(f)$.

If $f \in J$, then $\operatorname{ord}(f) \geq 2$. The lower degree form ideal $\operatorname{ldf}(J)$ associated to $J$ is

$$
\operatorname{ldf}(J):=(\operatorname{ldf}(f) \mid f \in J) \subseteq S[n]
$$

We have $\operatorname{ldf}(\operatorname{Ann}(M))=\operatorname{Ann}(\operatorname{tdf}(M))$ (see [9, Proposition 3], see also [7, formulas (2) and (3)]), whence

$$
\operatorname{gr}(S[n] / \operatorname{Ann}(M)) \cong S[n] / \operatorname{ldf}(\operatorname{Ann}(M)) \cong S[n] / \operatorname{Ann}(\operatorname{tdf}(M))
$$

Thus,

$$
H_{S[n] / \operatorname{Ann}(M)}(q)=\operatorname{dim}_{k}\left(\operatorname{tdf}(M)_{q}\right) .
$$

The module $M$ is said to be non-degenerate if $H_{S[n] / \operatorname{Ann}(M)}(1)=$ $\operatorname{dim}_{k}\left(\operatorname{tdf}(M)_{1}\right)=n$, i.e., if and only if the classes of $y_{1}, \ldots, y_{n}$ are in $\operatorname{tdf}(M)$. If $M=\langle F\rangle_{S[n]}$, then we write $\operatorname{tdf}(F)$ instead of $\operatorname{tdf}(M)$.

Let $A$ be Gorenstein with $s:=\operatorname{sdeg}(A)$, so that $\operatorname{Soc}(A)=\mathfrak{M}^{s} \cong k$. In particular, $A \cong S[n] / \operatorname{Ann}(F)$, where $F:=\sum_{i=0}^{s} F_{i}, F_{i} \in P[n]_{i}$. For each $h \geq 0$ we set

$$
F_{\geq h}:=\sum_{i=h}^{s} F_{i}
$$

(hence, $F_{s}=F_{\geq s}$ ).

Trivially, if $s \geq 1$, we can always assume that the homogeneous part of $F$ of degree 0 vanishes, i.e., $F=F_{\geq 1}$. Moreover, due to [6, Lemma $2.2]$, we know that if $s \geq 2$ and $\operatorname{Ann}(F) \subseteq S[n]_{+}^{2}$, then we can also assume $F_{1}=0$, i.e., $F=F_{\geq 2}$; we will always make such an assumption in what follows. 
We have a filtration with proper ideals (see [12, Definition 1.3]) of $\operatorname{gr}(A) \cong S[n] / \operatorname{ldf}(\operatorname{Ann}(F))$ :

$$
C_{A}(0):=\operatorname{gr}(A) \supset C_{A}(1) \supseteq C_{A}(2) \supseteq \cdots \supseteq C_{A}(s-2) \supseteq C_{A}(s-1):=0 .
$$

Via the epimorphism $S[n] \rightarrow \operatorname{gr}(A)$ we obtain an induced filtration

$$
\widehat{C}_{A}(0) \supset \widehat{C}_{A}(1) \supseteq \widehat{C}_{A}(2) \supseteq \cdots \supseteq \widehat{C}_{A}(s-2) \supseteq \widehat{C}_{A}(s-1) .
$$

By definition $\widehat{C}_{A}(0)=S[n]$ and $\widehat{C}_{A}(s-1)=\operatorname{ldf}(\operatorname{Ann}(F))$.

The quotients $Q_{A}(a):=C_{A}(a) / C_{A}(a+1) \cong \widehat{C}_{A}(a) / \widehat{C}_{A}(a+1)$ are reflexively graded $\operatorname{gr}(A)$-modules whose Hilbert function is symmetric around $(s-a) / 2$. In general, $\operatorname{gr}(A)$ is no longer Gorenstein, but the first quotient

$$
G(A):=Q_{A}(0) \cong S[n] / \operatorname{Ann}\left(F_{s}\right)
$$

is characterized by the property of being the unique (up to isomorphism) graded Gorenstein quotient $k$-algebra of $\operatorname{gr}(A)$ with the same socle degree. Moreover, the Hilbert function of $A$ satisfies

$$
H_{A}(i)=H_{\operatorname{gr}(A)}(i)=\sum_{a=0}^{s-2} H_{Q_{A}(a)}(i), \quad i \geq 0 .
$$

Since $H_{A}(0)=H_{G(A)}(0)=1$, it follows that, if $a \geq 1$, then $Q_{A}(a)_{0}=0$, whence $Q_{A}(a)_{i}=0$ when $i \geq s-a($ see $[\mathbf{1 2}$, Theorem 1.5]) for the same values of $a$. It follows that

$$
H_{A}(i)= \begin{cases}H_{Q_{A}(0)}(i) & \text { if } i=0, s \\ \sum_{a=0}^{s-i-1} H_{Q_{A}(a)}(i) & \text { if } 1 \leq i \leq s-1 .\end{cases}
$$

Moreover,

$$
H_{\operatorname{gr}(A) / C_{A}(a+1)}(i)=H_{S[n] / \hat{C}_{A}(a+1)}(i)=\sum_{\alpha=0}^{a} H_{Q_{A}(\alpha)}(i), \quad i \geq 0
$$

We set

$$
\begin{aligned}
f_{h} & :=\sum_{\alpha=0}^{s-h} H_{Q_{A}(\alpha)}(1)=H_{S[n] / \hat{C}_{A}(s-h+1)}(1) \\
& =H_{\operatorname{gr}(A) / C_{A}(s-h+1)}(1)
\end{aligned}
$$


(so that $\left.n=H_{A}(1)=f_{2}\right)$.

Finally, we introduce the following new invariant.

Definition 2.1. Let $A$ be a local, Artinian $k$-algebra with maximal ideal $\mathfrak{M}$ and $s:=\operatorname{sdeg}(A)$. The capital degree of $A$, denoted by $\operatorname{cdeg}(A)$, is defined to be the maximum integer $i$, if any, such that $H_{A}(i)>1$, and 0 otherwise. If $c=\operatorname{cdeg}(A)$, we also say that $A$ is a $c$ stretched $k$-algebra (for short, stretched if $c \leq 1$ ).

By definition $\operatorname{cdeg}(A) \geq 0$ and $\operatorname{cdeg}(A) \leq \operatorname{sdeg}(A)$, if $A$ is Gorenstein, then we also have $\operatorname{cdeg}(A)<\operatorname{sdeg}(A)$.

The following result, proved in [6, Lemma 4.1 and Remark 4.2], will be repeatedly used in the paper.

Lemma 2.2. Let $A$ be a local, Artinian, Gorenstein, $c$ stretched $k$ algebra. If $n:=H_{A}(1), m:=H_{A}(2), p:=H_{A}(c), s:=\operatorname{sdeg}(A)$, then

$$
A \cong S[n] / \operatorname{Ann}(F) \text {, }
$$

where

$$
\begin{gathered}
F:=y_{1}^{s}+\sum_{i=2}^{c+1} F_{i}+\sum_{j=m+1}^{n} y_{j}^{2}, \\
F_{i} \in P\left[f_{i}\right]_{i}, \quad i \geq 3, \quad F_{2} \in P\left[f_{3}\right]_{2}, \\
x_{1}^{c} \circ F_{c+1}=x_{1}^{i} \circ F_{i}=0, \quad i=3, \ldots, c+1
\end{gathered}
$$

and

$$
x_{2} \circ F_{c+1}, \ldots, x_{p} \circ F_{c+1}
$$

are linearly independent.

The rationality of the Poincaré series $P_{A}$ of every stretched ring $A$ is proved in [14]. The proof has been generalized to rings with $H_{A}(2)=2$ in [8] and to rings with $H_{A}(2)=3$ and $H_{A}(3)=1$ in [5]. The rationality of $P_{A}$ when $A$ is a 2 stretched $k$-algebra has been studied in [4] with the restriction $\operatorname{sdeg}(A)=3$. 
3. Decomposition of the apolar ideal. In the present section, we explain how to decompose the ideal $\operatorname{Ann}(F)$ as the sum of two simpler ideals. Such a decomposition will be used in the next section in order to reduce the calculation of the Poincaré series of $A$ to the one of a simpler $k$-algebra.

Lemma 3.1. Let $m<n, G \in P[m], H \in k\left[y_{m+1}, \ldots, y_{n}\right]$ be non-zero and $F=G+H$. Let us denote by $\operatorname{Ann}(G)$ and $\operatorname{Ann}(H)$ the annihilators of $G$ and $H$ inside $S[m]$ and $k\left[\left[x_{m+1}, \ldots, x_{n}\right]\right]$, respectively. Then,

$$
\operatorname{Ann}(F)=\operatorname{Ann}(G) S[n]+\operatorname{Ann}(H) S[n]+\left(\sigma_{G}-\sigma_{H}, x_{i} x_{j}\right)_{\substack{1 \leq i \leq m \\ m+1 \leq j \leq n}} .
$$

where $\sigma_{G} \in S[m]$ and $\sigma_{H} \in k\left[\left[x_{m+1}, \ldots, x_{n}\right]\right]$ are any series of order $\operatorname{deg}(G)$ and $\operatorname{deg}(H)$ such that $\sigma_{G} \circ G=\sigma_{H} \circ H=1$.

Proof. The inclusions $\operatorname{Ann}(G) S[n], \operatorname{Ann}(H) S[n] \subseteq \operatorname{Ann}(F)$ are completely trivial. Also, the inclusion

$$
\left(\sigma_{G}-\sigma_{H}, x_{i} x_{j}\right)_{\substack{1 \leq i \leq m \\ m+1 \leq j \leq n}} \subseteq \operatorname{Ann}(F)
$$

is easy to check. Thus,

$$
\operatorname{Ann}(G) S[n]+\operatorname{Ann}(H) S[n]+\left(\sigma_{G}-\sigma_{H}, x_{i} x_{j}\right)_{\substack{1 \leq i \leq m \\ m+1 \leq j \leq n}} \subseteq \operatorname{Ann}(F) .
$$

Conversely, let $p \in \operatorname{Ann}(F)$. Grouping the different monomials in $p$, we can write a decomposition $p=p_{\leq m}+p_{>m}+p_{\text {mix }}$, where $p_{\leq m} \in S[m]$, $p_{>m} \in k\left[\left[x_{m+1}, \ldots, x_{n}\right]\right]$ and, finally, $p_{\text {mix }} \in\left(x_{i} x_{j}\right)_{1 \leq i \leq m, m+1 \leq j \leq n} \subseteq$ $S[n]$.

It is clear that

$$
p_{\text {mix }} \in\left(\sigma_{G}-\sigma_{H}, x_{i} x_{j}\right)_{\substack{1 \leq i \leq m \\ m+1 \leq j \leq n}} \subseteq \operatorname{Ann}(F),
$$

hence it suffices to prove that

$$
p_{\leq m}+p_{>m} \in \operatorname{Ann}(G) S[n]+\operatorname{Ann}(H) S[n]+\left(\sigma_{G}-\sigma_{H}, x_{i} x_{j}\right)_{\substack{1 \leq i \leq m \\ m+1 \leq j \leq n}} .
$$

For this purpose, recall that $p_{\leq m}+p_{>m}=p-p_{\text {mix }} \in \operatorname{Ann}(F)$; thus, by definition,

$$
0=\left(p_{\leq m}+p_{>m}\right) \circ F=p_{\leq m} \circ G+p_{>m} \circ H .
$$


Hence,

$$
p_{\leq m} \circ G=u=-p_{>m} \circ H .
$$

Since $p_{\leq m} \circ G \in P[m]$ and $p_{>m} \circ H \in k\left[y_{m+1}, \ldots, y_{n}\right]$, it follows that $u \in k$. So $p_{\leq m}-u\left(\sigma_{G}-\sigma_{H}\right) \in \operatorname{Ann}(G) S[n]$, whence

$$
\begin{aligned}
p_{\leq m} \in\left(\sigma_{G}-\sigma_{H}\right)+\operatorname{Ann}(G) S[n] \subseteq \operatorname{Ann}(G) S[n]+\operatorname{Ann}(H) S[n] \\
+\left(\sigma_{G}-\sigma_{H}, x_{i} x_{j}\right)_{\substack{1 \leq i \leq m \\
m+1 \leq j \leq n}} .
\end{aligned}
$$

A similar argument shows that

$$
\begin{aligned}
p_{>m} \in\left(\sigma_{G}-\sigma_{H}\right)+\operatorname{Ann}(H) S[n] \subseteq & \operatorname{Ann}(G) S[n]+\operatorname{Ann}(H) S[n] \\
& +\left(\sigma_{G}-\sigma_{H}, x_{i} x_{j}\right)_{\substack{1 \leq i \leq m \\
m+1 \leq j \leq n}},
\end{aligned}
$$

and this concludes the proof.

Let $F$ be as in the statement above. Then Lemma 3.1 with

$$
G:=\sum_{i=2}^{s} F_{i}, \quad H:=\sum_{j=m+1}^{n} y_{j}^{2}
$$

yield the following corollary.

Corollary 3.2. Let $m<n, G \in P[m]$ be non-zero, and let

$$
F=G+\sum_{j=m+1}^{n} y_{j}^{2}
$$

Let us denote by $\operatorname{Ann}(G)$ the annihilator of $G$ inside $S[m]$. Then

$$
\operatorname{Ann}(F)=\operatorname{Ann}(G) S[n]+\left(x_{j}^{2}-2 \sigma, x_{i} x_{j}\right)_{\substack{1 \leq i<j \leq n \\ j \geq m+1}}
$$

where $\sigma \in S[m]$ has order $\operatorname{deg}(G)$ and $\sigma \circ G=1$.

Proof. It suffices to apply Lemma 3.1 with

$$
H:=\sum_{j=m+1}^{n} y_{j}^{2},
$$


taking into account that

$$
\operatorname{Ann}(H)=\left(x_{j}^{2}-x_{m+1}^{2}, x_{i} x_{j}\right)_{\substack{m+1 \leq i<j \leq n \\ j \geq m+1}},
$$

and that $x_{m+1}^{2} \circ H=2$.

4. Rationality of Poincaré series. We now focus on the Poincaré series $P_{A}(z)$ of the $k$-algebra $A$ defined in the introduction: we will generalize the aforementioned classical results $[\mathbf{5}, \mathbf{8}, \mathbf{1 4}]$.

Proposition 4.1. Let $A$ be a local, Artinian, Gorenstein, k-algebra with $n=H_{A}(1)$. Assume $A \cong S[n] / \operatorname{Ann}(F)$ where

$$
F=F_{\geq 2}:=G+\sum_{j=m+1}^{n} y_{j}^{2}
$$

for a suitable non-zero $G \in P[m]$. Then

$$
P_{A}(z)=\frac{P_{B}(z)}{1-\left(H_{A}(1)-H_{B}(1)\right) z P_{B}(z)},
$$

where $B:=S[m] / \operatorname{Ann}(G)$.

Proof. Since $F=F_{\geq 2}$, it follows that the same is true for $G$; hence, $m=H_{B}(1)$. Now we can use Corollary 3.2 above.

Besides the decomposition result proved in the previous section, we will also use the two following fundamental facts about Poincaré series:

- for each local Artinian, Gorenstein $\operatorname{ring} C$ with $\operatorname{emdim}(C) \geq 2$,

$$
P_{C}(z)=\frac{P_{C / \operatorname{Soc}(C)}(z)}{1+z^{2} P_{C / \operatorname{Soc}(C)}(z)}
$$

(see [2, Theorem 2]);

- for each local Artinian ring $C$ with maximal ideal $\mathfrak{N}$ and linearly independent elements $c_{1}, \ldots, c_{h} \in \mathfrak{N} \backslash \mathfrak{N}^{2}$ of $\operatorname{Soc}(C)$, then

$$
P_{C}(z)=\frac{P_{C /\left(c_{1}, \ldots, c_{h}\right)}(z)}{1-h z P_{C /\left(c_{1}, \ldots, c_{h}\right)}(z)}
$$

(see [11, Proposition 3.4.4]). 
Due to Corollary 3.2, we have

$$
\operatorname{Ann}(F)+\left(\sigma, x_{m+1}, \ldots, x_{n}\right)=\operatorname{Ann}(G) S[n]+\left(\sigma, x_{m+1}, \ldots, x_{n}\right),
$$

where $\sigma$ is as in Corollary 3.2. Thus,

$$
\frac{S[n]}{\operatorname{Ann}(F)+\left(\sigma, x_{m+1}, \ldots, x_{n}\right)} \cong \frac{S[m]}{\operatorname{Ann}(G)+(\sigma)} .
$$

Trivially, $S[m] / \operatorname{Ann}(G)$ is a local, Artinian, Gorenstein, $k$-algebra.

On the one hand, we know that $\operatorname{Soc}(A)$ is principal because $A$ is Gorenstein. On the other hand, $\sigma \notin \operatorname{Ann}(F)$, because $\sigma \in S[m]$, whence $\sigma \circ F=\sigma \circ G=1$, and $x_{i} \sigma \in \operatorname{Ann}(F)$ for each $i=1, \ldots, n$, because $x_{i} \sigma \circ F=0$. It follows that the class of $\sigma$ generates $\operatorname{Soc}(A)$. Formula (4.1) implies that

$$
P_{A}(z)=\frac{P_{S[n] / \operatorname{Ann}(F)+(\sigma)}(z)}{1+z^{2} P_{S[n] / \operatorname{Ann}(F)+(\sigma)}(z)} .
$$

Notice that

$$
x_{i} x_{j} \in \operatorname{Ann}(F)+(\sigma), \quad i=1, \ldots, n, j=m+1, \ldots, n, i \leq j .
$$

In particular, $x_{m+1}, \ldots, x_{n} \in \operatorname{Soc}(S[n] / \operatorname{Ann}(F)+(\sigma))$. It follows from formula (4.2) that

$$
P_{S[n] / \operatorname{Ann}(F)+(\sigma)}(z)=\frac{P_{S[n] / \operatorname{Ann}(F)+\left(\sigma, x_{m+1}, \ldots, x_{n}\right)}(z)}{1-(n-m) z P_{S[n] / \operatorname{Ann}(F)+\left(\sigma, x_{m+1}, \ldots, x_{n}\right)}(z)} .
$$

The inverse formula of (4.1) finally yields

$P_{S[n] / \operatorname{Ann}(F)+\left(\sigma, x_{m+1}, \ldots, x_{n}\right)}=P_{S[m] / \operatorname{Ann}(G)+(\sigma)}(z)=\frac{P_{S[m] / \operatorname{Ann}(G)}(z)}{1-z^{2} P_{S[m] / \operatorname{Ann}(G)}(z)}$.

The statement now follows by combining the three equalities above.

A first immediate consequence of Proposition 4.1 is the following corollary.

Corollary 4.2. Let $A$ be a local, Artinian, Gorenstein, k-algebra with $n=H_{A}(1)$. Assume that $A:=S[n] / \operatorname{Ann}(F)$, where

$$
F=F_{\geq 2}:=G+\sum_{j=m+1}^{n} y_{j}^{2},
$$


for a suitable $G \in P[m]$. Then the series $P_{A}(z)$ is rational if and only if the same is true for $P_{B}(z)$ where $B:=S[m] / \operatorname{Ann}(G)$.

Proof. If $G \neq 0$, then the result follows from Proposition 4.1 above.

If $G=0$, then $A$ is stretched; thus, $P_{A}(z)$ is rational by [14, Theorem 2]. Moreover, in this case, $B=0$; thus, $P_{B}(z)=0$ is also rational and the equivalence holds.

Corollary 4.3. Let $A$ be a local, Artinian, Gorenstein, k-algebra with $n=H_{A}(1)$. Assume that $A:=S[n] / \operatorname{Ann}(F)$ where

$$
F=F_{\geq 2}:=G+\sum_{j=m+1}^{n} y_{j}^{2}
$$

for a suitable $G \in P[4]$. Then $P_{A}(z)$ is rational.

Proof. The statement follows from rationality of the Poincaré series of each local Artinian, Gorenstein ring with embedding dimension at most four (see $[\mathbf{1 3}, \mathbf{1 6}, \mathbf{1 7}]$ ).

Let $A$ be a local, Artinian, Gorenstein $k$-algebra with $n:=H_{A}(1)$. Recall that we defined the numbers $f_{h}=H_{\operatorname{gr}(A) / C_{A}(s-h+1)}(1)$ in Section 2.

Corollary 4.4. Let $A$ be a local, Artinian, Gorenstein k-algebra such that $f_{3} \leq 4$. Then $P_{A}(z)$ is rational.

Proof. The statement follows from Corollary 4.3, taking into account Lemma 2.2.

5. Examples of algebras with rational Poincaré series. In this section, we give some examples of local, Artinian, Gorenstein $k$-algebras $A$ with rational $P_{A}$ using the results proved in the previous section and some other classical results that we now go to quickly recall.

Remark 5.1. For the following results, we refer to [3, Chapter 4]. 
If $i_{0} \in \mathbb{Z}$ is positive, then each positive $a \in \mathbb{Z}$ can be uniquely written in the form

$$
a=\sum_{i=1}^{i_{0}}\left(\begin{array}{c}
k(i) \\
i
\end{array}\right)
$$

where $0 \leq k(i-1)<k(i)$ (see [3, Lemma 4.2.6]). We define

$$
a^{\left\langle i_{0}\right\rangle}:=\sum_{i=1}^{i_{0}}\left(\begin{array}{c}
k(i)+1 \\
i+1
\end{array}\right) .
$$

Macaulay's growth theorem (see [3, Theorem 4.2.10]) states that the following conditions are equivalent for a function $h: \mathbb{N} \rightarrow \mathbb{N}$ :

- there exists a graded $k$-algebra $R$ with Hilbert function $H_{R}=$ $h$;

- one has $h(0)=1$ and $h(i+1) \leq h(i)^{\langle i\rangle}$ for each $i \geq 1$.

An immediate consequence of the above equivalence is the following. Let $A$ be a local $k$-algebra. If $H_{A}(i) \leq i$ for some $i$, then $H_{A}(j) \leq H_{A}(i)$ for each $j \geq i$. Such a remark will be used intensively in the following proofs.

The following lemma generalizes a result due to Stanley (see [15, Section II.6 (c)]).

Lemma 5.2. Let $A$ be a local, Artinian, Gorenstein, 3 stretched $k$ algebra. If $H_{A}(3) \leq 5$, then

$$
\sum_{a=0}^{s-4} H_{Q_{A}(a)}(2) \geq H_{A}(3) .
$$

Proof. We set $m:=H_{A}(3)$ and

$$
p:=\sum_{a=0}^{s-4} H_{Q_{A}(a)}(2) .
$$

We have to show that $p \geq m$ : assume $p \leq m-1$. 
If $s=4$, then

$$
H_{Q_{A}(0)}=\sum_{a=0}^{s-4} H_{Q_{A}(a)}
$$

In particular, such a sum is the Hilbert function of a graded Gorenstein $k$-algebra; thus, it must be symmetric, say $(1, m, p, m, 1)$.

Let $s \geq 5$. Then formula (2.4) for $i \geq 4$ implies

$$
1=H_{A}(i)=\sum_{a=0}^{s-5} H_{Q_{A}(a)}(i) .
$$

Since $A$ is 3 -stretched and $H_{Q_{A}(0)}(i) \geq 1$ for $i \leq s$, it follows that $H_{Q_{A}(0)}(1)=H_{Q_{A}(a)}(s-1)=1$. Thus, Remark 5.1 implies $H_{Q_{A}(0)}(i)=$ 1 for $i \leq s$.

The same argument also proves $H_{Q_{A}(a)}(1)=H_{Q_{A}(a)}(s-a-1)=0$ in the range $1 \leq a \leq s-5$. Due to formula (2.5) we deduce that

$$
H_{\operatorname{gr}(A) / C_{A}(s-4)}(1)=\sum_{\alpha=0}^{s-5} H_{Q_{A}(\alpha)}(1)=1
$$

whence

$$
H_{\operatorname{gr}(A) / C_{A}(s-4)}(i)=\sum_{\alpha=0}^{s-5} H_{Q_{A}(\alpha)}(i)=1 \quad \text { for } i \leq s .
$$

We conclude that

$$
H_{Q_{A}(a)}= \begin{cases}(1,1,1,1,1, \ldots, 1) & \text { if } a=0 \\ (0,0,0,0,0, \ldots, 0) & \text { if } a=1, \ldots, s-5 \\ (0, m-1, p-1, m-1,0, \ldots, 0) & \text { if } a=s-4\end{cases}
$$

In particular,

$$
\sum_{a=0}^{s-4} H_{Q_{A}(a)}=H_{Q_{A}(0)}+H_{Q_{A}(s-4)} .
$$

Notice that $f_{4}=m$. 
Recall that we have assumed $p<m$. Formula (2.5) and Remark 5.1 imply that if

$$
p=H_{\operatorname{gr}(A) / C_{A}(s-4)}(2)=\sum_{a=0}^{s-4} H_{Q_{A}(a)}(2) \leq 2,
$$

then $m=H_{\operatorname{gr}(A) / C_{A}(s-4)}(3) \leq p$, contradicting our assumption $p \leq$ $m-1$. We conclude that $3 \leq p$ necessarily. Moreover, if $p=3$, then the bound of Macaulay's growth theorem (see Remark 5.1) gives $m \leq 4$. Since we assumed $m \leq 5$, we are left with two cases: $p=3$ and $m=4$ or $p=4$ and $m=5$. We examine the second case, the first one being analogous.

Lemma 2.2 with $c=3$ asserts the existence of a polynomial $F:=$ $y_{1}^{s}+F_{4}+F_{3}+F_{2}$ such that $F_{i} \in P\left[f_{i}\right]_{i}, x_{1}^{3} \circ F_{4}=0$, the derivatives $x_{2} \circ F_{4}, x_{3} \circ F_{4}, x_{4} \circ F_{4}$ and $x_{5} \circ F_{4}$ are linearly independent and $A \cong S[n] / \operatorname{Ann}(F)$. We set $B:=S[n] / \operatorname{Ann}\left(F_{\geq 4}\right)$.

We first check that

$$
H_{B}=\sum_{a=0}^{s-4} H_{Q_{A}(a)}=(1,5,4,5,1, \ldots, 1) .
$$

On the one hand, [12, Lemma 1.10] implies that $\widehat{C}_{A}(a)=\widehat{C}_{B}(a)$, $a \leq s-3$, whence

$$
H_{B}(1) \geq \sum_{a=0}^{s-4} H_{Q_{B}(a)}(1)=\sum_{a=0}^{s-4} H_{Q_{A}(a)}(1)=5 .
$$

On the other hand, $F_{\geq 4} \in P\left[f_{4}\right]=P[5]$, whence $5=H_{B}(1) \leq 5$. It follows that equality holds; thus, $H_{Q_{B}(s-2)}(1)=H_{Q_{B}(s-3)}(1)=0$. By symmetry, we finally obtain $H_{Q_{B}(s-2)}=H_{Q_{B}(s-3)}=0$. This last vanishing completes the proof of the equality

$$
H_{B}=\sum_{a=0}^{s-4} H_{Q_{A}(a)}=(1,5,4,5,1, \ldots, 1) .
$$

Let $I \subseteq k\left[x_{1}, \ldots, x_{n}\right] \subseteq S[n]$ be the ideal generated by the forms of degree at most 2 inside $\operatorname{Ann}\left(\operatorname{tdf}\left(F_{\geq 4}\right)\right)=\operatorname{ldf}\left(\operatorname{Ann}\left(F_{\geq 4}\right)\right)$. We obviously have $x_{6}, \ldots, x_{n} \in I$, because $F_{\geq 4} \in P[5]$. 
Let

$$
I^{\text {sat }}:=\bigcup_{h=1}^{\infty}\left(I:\left(x_{1}, \ldots, x_{n}\right)^{h}\right):
$$

it is easy to check that $I^{\text {sat }}$ is an ideal. We set $R:=k\left[x_{1}, \ldots, x_{n}\right] / I$, $R^{\text {sat }}:=k\left[x_{1}, \ldots, x_{n}\right] / I^{\text {sat }}$. Due to the definition of $I$ we know that $H_{R}(t) \geq H_{B}(t)$ for each $t \geq 0$, and equality holds true for $t \leq 2$. Moreover, we know that

$$
H_{B}(2)^{\langle 2\rangle}=H_{B}(3) \leq H_{R}(3) \leq H_{R}(2)^{\langle 2\rangle}=H_{B}(2)^{\langle 2\rangle} ;
$$

hence,

$$
H_{R}(3)=\left(\begin{array}{l}
4 \\
3
\end{array}\right)+\left(\begin{array}{l}
2 \\
2
\end{array}\right)=H_{R}(2)^{\langle 2\rangle}
$$

The Gotzmann persistence theorem (see [3, Theorem 4.3.3]) implies that

$$
H_{R}(t)=\left(\begin{array}{c}
t+1 \\
t
\end{array}\right)+\left(\begin{array}{c}
t-1 \\
t-1
\end{array}\right)=t+2, \quad t \geq 2 .
$$

We infer $H_{R^{\text {sat }}}(t)=t+2, t \gg 0$.

When saturating, the ideal can only increase its size in each degree; hence, $H_{R^{\text {sat }}}(t) \leq H_{R}(t)$ for each $t \geq 0$. Again Macaulay's bound (see Remark 5.1) thus forces $H_{R^{\text {sat }}}(t)=H_{R}(t)=t+2$ for $t \geq 2$. In particular, the components $I_{t}$ and $I_{t}^{\text {sat }}$ of degree $t \geq 2$ of $I$ and $I^{\text {sat }}$ coincide.

Since $H_{R^{\text {sat }}}$ is non-decreasing, it follows that

$$
H_{R^{\text {sat }}}(1) \leq H_{R^{\text {sat }}}(2)=4<5=H_{B}(1)=H_{R}(1) .
$$

In particular, there exists a non-zero linear form $\ell \in I^{\text {sat }} \backslash I$. The equality $I_{2}=I_{2}^{\text {sat }}$ forces $\ell x_{j} \in I_{2} \subseteq \operatorname{Ann}\left(\operatorname{tdf}\left(F_{\geq 4}\right)\right), j=1, \ldots, n$. Since $x_{6}, \ldots, x_{n} \in I$, it follows that we can assume $\ell \in S[5] \subseteq S[n]$, i.e.,

$$
\ell=\sum_{i=1}^{5} \ell_{i} x_{i}, \quad \ell_{i} \in k
$$

Moreover, we also know that $y_{1}^{s} \in \operatorname{tdf}\left(F_{\geq 4}\right)$ because $s \geq 5$. Hence, the condition $\ell x_{1} \in \operatorname{Ann}\left(\operatorname{tdf}\left(F_{\geq 4}\right)\right)$ implies $\bar{\ell}_{1}=0$ and at least one of the remaining coefficients is non-zero. 
If $j \geq 2$, then $x_{j} \circ F_{\geq 4}=x_{j} \circ F_{4}$, thus the condition $x_{j} \ell \in$ $I_{2} \subseteq \operatorname{Ann}\left(\operatorname{tdf}\left(F_{\geq 4}\right)\right)$ implies that $\ell \circ F_{4}$ does not depend on such $x_{j}$, $j=2, \ldots, 5$. It follows that $\ell \circ F_{4}$ is a multiple of $y_{1}^{3}$. Since we know that $x_{1}^{3} \circ F_{4}=0$, we conclude that $\ell \circ F_{4}=0$. Such a vanishing contradicts the linear independence of the derivatives

$$
x_{2} \circ F_{4}, \quad x_{3} \circ F_{4}, \quad x_{4} \circ F_{4}, \quad x_{5} \circ F_{4} .
$$

The proof of the statement is now complete.

Using the results proved in the previous section and Lemma 5.2 we are able to handle the first example of this section, proving the following theorem generalizing [4, Corollary 2.2].

Theorem 5.3. Let $A$ be a local, Artinian, Gorenstein k-algebra with $H_{A}(2) \leq 4$ and $\operatorname{cdeg}(A) \leq 3$. Then $P_{A}$ is rational.

Proof. Let us examine the case $\operatorname{cdeg}(A)=3$, the other ones being similar, but simpler. The proofs of these cases are left to the reader. Lemma 5.2 yields:

$$
H_{A}(2) \geq \sum_{a=0}^{s-4} H_{Q(a)}(2) \geq H_{A}(3) .
$$

If $\operatorname{sdeg}(A) \geq 5$, then decomposition (2.3) is

$$
(1,1, \ldots, 1)+\left(0, a_{1}, a_{2}, a_{1}, 0\right)+\left(0, b_{1}, b_{1}, 0\right)+\left(0, c_{1}, 0\right)
$$

for some integers $a_{1}, a_{2}, b_{1}, c_{1}$. Inequality (5.1) is equivalent to $a_{1} \leq a_{2}$. We know that $H_{A}(2)=a_{2}+b_{1}+1 \leq 4$, so $f_{3}=a_{1}+b_{1}+1 \leq 4$, and the argument follows from Corollary 4.4. In the case $\operatorname{sdeg}(A)=4$, the decomposition (2.3) changes, but the argument stays the same.

Now we skip the condition $\operatorname{cdeg}(A)=3$, but we impose a restriction on the shape of $H_{A}$. The following theorem generalizes a well-known result proved when either $m=1,2$ (see $[\mathbf{8 , 1 4 ]}$ ) or $m \leq 4$ and $s=3$ (see again [4]).

Theorem 5.4. Let $A$ be a local, Artinian, Gorenstein k-algebra such that $H_{A}(i)=m, 2 \leq i \leq \operatorname{cdeg}(A)$. If $m \leq 4$, then $P_{A}$ is rational. 
Proof. Let $c:=\operatorname{cdeg}(A), n:=H_{A}(1)$, take a polynomial $F:=y_{1}^{s}+$ $F_{c+1}+\cdots, F_{c+1} \in P\left[f_{c+1}\right]_{c+1}=P[m]_{c+1}$ such that $A \cong S[n] / \operatorname{Ann}(F)$ (see Lemma 2.2) and set $B:=S[n] / \operatorname{Ann}\left(F_{\geq c+1}\right)$ so that $Q_{A}(a)=$ $Q_{B}(a)$ for $a \leq s-c-1$ (again by [12, Lemma 1.10]). In particular, $H_{B}(c)=m$, thus decomposition (2.3) implies $H_{B}(1) \geq m$. Since we know that $F_{\geq c+1} \in P[m]$, it follows that $H_{B}(1) \leq m$, hence equality must hold.

As in the proof of the previous lemma one immediately checks that either $s=c+1$ and $H_{Q_{A}(0)}=(1, m, \ldots, m, 1)$, or $s \geq c+2$ and

$$
H_{Q_{A}(a)}= \begin{cases}(1,1, \ldots, 1,1, \ldots, 1) & \text { if } a=0 \\ (0,0, \ldots, 0,0, \ldots, 0) & \text { if } a=1, \ldots, s-c-2, \\ (0, m-1, \ldots, m-1,0, \ldots, 0) & \text { if } a=s-c-1\end{cases}
$$

Assume that $H_{B}(i) \leq m-1 \leq 3$ for some $i=2, \ldots, c-1$. Let $i_{0}$ be the maximal of such $i$ 's. If $i_{0} \geq 3$, then $H_{B}\left(i_{0}\right) \leq i_{0}<m$ so by Remark 5.1, we get that $H_{B}(i)<m$ for all $i>i_{0}$. In particular, $H_{B}(c)<m$, which is a contradiction. We conclude that $i_{0}=2$.

Due to the symmetry of $H_{Q_{B}(s-c-1)}$, we deduce that $c=3$. If $H_{Q_{B}(s-3)}(2)=q$, the symmetry of $H_{Q_{B}(s-3)}$ implies $H_{Q_{B}(s-3)}(1)=q$; hence decomposition (2.3) implies

$$
m=H_{B}(1)=\sum_{a=0}^{s-2} H_{Q_{B}(a)}(1)=m+q+H_{Q_{B}(s-2)}(1) .
$$

It follows that $q=H_{Q_{B}(s-2)}(1)=0$, whence $H_{B}=(1, m, p, m, 1, \ldots, 1)$ where $p \leq m-1$ which cannot occur by Lemma 5.2.

We conclude that $H_{Q_{A}(s-c-1)}(i)=H_{Q_{B}(s-c-1)}(i)=m-1$ for each $i=2, \ldots, c$, then the hypothesis on $H_{A}(i)$ and decomposition $(2.3)$ yield

$$
H_{Q_{A}(a)}= \begin{cases}(0,0,0, \ldots, 0,0, \ldots, 0) & \text { if } a=s-c, \ldots, s-3 \\ (0, n-m, 0, \ldots, 0,0, \ldots, 0) & \text { if } a=s-2\end{cases}
$$

whence

$$
f_{3}=\sum_{a=1}^{s-3} H_{Q(a)}(1)=m \leq 4
$$


As a third example, we skip the condition on the shape of $H_{A}$ but we put a limit on $\operatorname{dim}_{k}(A)$, slightly extending the result proved in [6].

Theorem 5.5. Let $A$ be a local, Artinian, Gorenstein k-algebra with $\operatorname{dim}_{k}(A) \leq 16$ and $H_{A}(2) \leq 4$. Then $P_{A}$ is rational.

Proof. Due to [13], we can restrict our attention to $k$-algebras $A$ with $H_{A}(1) \geq 5$.

Rationality of the Poincare series of stretched $k$-algebras is proved in [14]. For $k$-algebras $A$ with $H_{A}(2)=2$, which are called almost stretched, see [8]. For the case of $k$-algebras $A$ with $\operatorname{sdeg}(A)=3$ and $H_{A}(2) \leq 4$, see [4]. Finally, the case $H_{A}(i)=m, 2 \leq i \leq \operatorname{cdeg}(A)$ with $m \leq 4$ is covered by Theorem 5.4 above.

There are several cases which are not covered by the aforementioned results. In each of these cases one can check that the condition $f_{3} \leq 4$ of Corollary 4.4 is fulfilled.

Due to the above summary of known results, we can assume $H_{A}(2) \geq 3$. The restriction $H_{A}(2) \leq 4$ implies $H_{A}(3) \leq 5$ again by Remark 5.1.

Theorem 5.3 deals with the case $\operatorname{sdeg}(A)=4$. Let us analyze the cases $\operatorname{sdeg}(A)=5$ and $\operatorname{dim}_{k} A \leq 16$. The decomposition is

$$
\left(1, a_{1}, a_{2}, a_{2}, a_{1}, 1\right)+\left(0, b_{1}, b_{2}, b_{1}, 0\right)+\left(0, c_{1}, c_{1}, 0\right)+\left(0, d_{1}, 0\right),
$$

for some integers $a_{1}, a_{2}, b_{1}, b_{2}, c_{1}, d_{1}$. If $a_{1}=1$, then the $k$ algebra is 3 -stretched, so we may suppose $a_{1} \geq 2$. We know that $H_{A}(2)=a_{2}+b_{2}+c_{1} \leq 4$, and we would like to prove $a_{1}+b_{1}+c_{1} \leq 4$. Suppose $a_{1}+b_{1}+c_{1} \geq 5$. Then the inequality on the dimension of $A$ shows that $2 \cdot a_{2}+b_{2} \leq 4$, in particular, $a_{2} \leq 2$ and from Remark 5.1, it follows that $a_{1}=a_{2}=2$. It follows that $b_{2}=0$ and once again, from the same remark, $b_{1}=0$. This forces $a_{1}+b_{1}+c_{1}=2+c_{1}=a_{2}+b_{2}+c_{1} \leq 4$, a contradiction.

Let us now suppose that $\operatorname{sdeg}(A)=6$. Look at the first row of the symmetric decomposition (2.3): $\left(1, a_{1}, a_{2}, a_{3}, a_{2}, a_{1}, 1\right)$.

- If $a_{1} \geq 3$, then $a_{2}, a_{3} \geq 3$ and the sum of the row is at least 17 .

- If $a_{1}=2$, then $a_{2}=a_{3}=2$ and the sum of the row is 12 . If we suppose that $f_{3} \geq 5$, then the sum of the first column of the 
remaining part of the decomposition will be at least 3 , so the sum of whole remaining part will be at least $2 \cdot 3=6$, and the dimension will be at least $12+6>16$.

- Suppose $a_{1}=1$, and look at the second row $\left(0, b_{1}, b_{2}, b_{2}, b_{1}, 0\right)$. If $b_{1}=0$, then the $k$-algebra is 3 -stretched so the result follows from Theorem 5.3. From $H_{A}(2) \leq 4$, it follows that $b_{2} \leq 3$. If $b_{2}=3$, then $b_{1} \geq 2$ so the dimension is at least $7+10>16$. If $b_{2} \leq 2$, then $b_{1} \leq b_{2}$ from Remark 5.1. Hence, the same argument as before applies.

Let us finally suppose that $\operatorname{sdeg}(A) \geq 7$. Take the first row, beginning with $\left(1, a_{1}, a_{2}, \ldots\right)$. If $a_{1} \geq 3$, then its sum is at least $3 \cdot \operatorname{sdeg}(A)-1>16$. If $a_{1}=2$, the sum of this row is $2 \cdot \operatorname{sdeg}(A) \geq 14$. Then one can argue as in the case $\operatorname{sdeg}(A)=6, a_{1}=2$. A similar reasoning shows that, when $a_{1}=1$, the $k$-algebra has decomposition $(1,1, \ldots, 1)+(0,4,4,0)$, and so $H_{A}(2) \geq 5$.

Acknowledgments. The authors are grateful to the anonymous referee whose comments, corrections and suggestions have considerably contributed to improve the exposition of the paper.

\section{REFERENCES}

1. L. Avramov, A. Kustin and M. Miller, Poincaré series of modules over local rings of small embedding codepth or small linking number, J. Algebra 118 (1988), 162-204.

2. L. Avramov and G. Levin, Factoring out the socle of a Gorenstein ring, J. Algebra 55 (1978), 74-83.

3. W. Bruns and J. Herzog, Cohen-Macaulay rings, II, Cambridge University Press, Cambridge, 1998.

4. G. Casnati, J. Elias, R. Notari and M.E. Rossi, Poincaré series and deformations of Gorenstein local algebras with low socle degree, Comm. Algebra 41 (2013), 1049-1059.

5. G. Casnati and R. Notari, The Poincaré series of a local Gorenstein ring of multiplicity up to 10 is rational, Proc. Indian Acad. Sci. Math. Sci. 119 (2009), 459-468.

6. A structure theorem for 2-stretched Gorenstein algebras, preprint arXiv: 1312.2191 [math.AC], J. Commutative Algebra, to appear.

7. J. Elias and M.E. Rossi, Isomorphism classes of short Gorenstein local rings via Macaulay's inverse system, Trans. Amer. Math. Soc. 364 (2012), 4589-4604.

8. J. Elias and G. Valla, A family of local rings with rational Poincaré series, Proc. Amer. Math. Soc. 137 (2009), 1175-1178. 
9. J. Emsalem, Géométrie des points épais, Bull. Soc. Math. France 106 (1978), 399-416.

10. R. Hartshorne, Algebraic geometry, Grad. Texts Math. 52, Springer, New York, 1977.

11. T.H. Gulliksen and G. Levin, Homology of local rings, Queen's Papers Pure Appl. Math. 20 (1969), x+192.

12. A.V. Iarrobino, Associated graded algebra of a Gorenstein Artin algebra, Mem. Amer. Math. Soc. 107 (1994), viii+115.

13. C. Jacobsson, A. Kustin and M. Miller, The Poincaré series of a codimension four Gorenstein ring is rational, J. Pure Appl. Algebra 38 (1985), 255-275.

14. J.D. Sally, The Poincaré series of stretched Cohen-Macaulay rings, Canad. J. Math. 32 (1980), 1261-1265.

15. R.P. Stanley, Combinatorics and commutative algebra, Birkhäuser, Berlin, 1983.

16. J. Tate, Homology of Noetherian rings and local rings, Illinois J. Math. 1 (1957), 14-25.

17. H. Wiebe, Über homologische Invarianten lokaler Ringe, Math. Ann. 179 (1969), 257-274.

Dipartimento di Scienze Matematiche, Politecnico di Torino, corso Duca DEgli Abruzzi 24, 10129 Torino, Italy

Email address: gianfranco.casnati@polito.it

Faculty of Mathematics, Informatics, and Mechanics, University of WarSaW, Banacha 2, 02-097 Warsaw, Poland

Email address: jjelisiejew@mimuw.edu.pl

Dipartimento di Matematica, Politecnico di Milano, via Bonardi 9, 20133 Milano, ItAaly

Email address: roberto.notari@polimi.it 